\title{
Quelles pratiques littéraciques pour les enfants sourds?
}

\section{Edyta Tominska}

Cet article décrit les pratiques littéraciques introduites dans une classe bilingue (LSF/français) pour enfants sourds. Un ancrage conceptuel des programmes bilingues dans l'éducation de ces enfants sera suivi par des apports d'études sur l'entrée dans l'écrit des jeunes enfants, et notamment, parmi d'autres, des microgenèses didactiques. Une séance de Lecture Interactive nous servira d'illustration pour relever des composantes émergentes du savoir littéracique dans le discours de la classe. Dans cette situation didactique complexe, nous appréhendons ces composantes activées grâce àldans l'interaction, aussi bien que les ajustements réciproques qu'accomplissent les enseignants et les élèves pour se (faire) comprendre, les supports visuels qu'ils utilisent, les rôles joués par les deux langues.

\section{Introduction}

Avant d'entrer dans le vif du sujet de notre contribution, nous proposons quelques résultats généraux de la recherche en surdité (ou Deaf Studies) concernant l'éducation des enfants sourds et malentendants ainsi que les transformations qui la régissent. Les données scientifiques établies aux Etats-Unis montrent un changement important dans l'éducation offerte à ces enfants: en 1966, plus de 80\% des enfants sourds fréquentaient des écoles spécialisées, et seulement $20 \%$ étaient intégrés dans les écoles publiques proches de leur domicile (mainstreamed education). En 2006, cette proportion est renversée (Marschark \& Hauser, 2008, p. 6). Ces changements impliquent l'évolution des besoins du terrain éducatif et scolaire qui accueillent ces enfants. D'une part, en matière des connaissances sur leur façon d'apprendre et de progresser, sur leurs besoins particuliers etc., les recherches actuelles démontrent que ces enfants ne sont pas «des entendants qui ne peuvent pas entendre» mais different dans leur fonctionnement cognitif, ce qui pourrait influencer leurs performances scolaires, leur formation ou leur carrière académique (Marschark, 2007). D’autre part, les 
transformations interrogent les pratiques éducatives/enseignantes à adopter, les moyens d'enseignement, les outils qui peuvent favoriser les apprentissages de cette population d'enfants, dans le cadre spécialisé ou inclusif. Ces changements posent, en troisième lieu, la question de la formation des enseignants. Cette formation devrait combler le manque d'information et de préparation de ces derniers, dans le but d'améliorer la prise en charge éducative/scolaire actuelle, en connaissance de cause et avec une meilleure compréhension de l'enfant sourd dans son développement (Marschark, Convertino \& LaRoch, 2006).

Aujourd'hui, les sourds américains atteignent cependant les écoles supérieures qui comptent 30'000 étudiants sourds dans les programmes post-obligatoires. Malheureusement, seulement un sur quatre de ces étudiants finalise ses études et obtient un diplôme. Des résultats comparables sont rapportés du Québec (Daigle, 2003, cité par Tièche Christinat \& CSPS, 2010). Quant aux pays européens, peu de sourds arrivent au niveau de baccalauréat, et rares sont ceux qui étudient et qui obtiennent des diplômes du niveau supérieur. Très peu de recherches en Europe rapportent les données globales, statistiques sur cette population de 0-20 ans et sur leur prise en charge éducative/scolaire. La Suisse partage ce manque de suivi dans sa globalité. C'est pour cela que l'Observatoire de la surdité en Suisse Romande et au Tessin est une grande avancée. Les données de cet Observatoire permettent une évaluation des besoins du terrain ainsi qu'une observation de l'évolution des enfants accueillis, dans la majorité des cas, dans les écoles ordinaires (Tièche Christinat \& CSPS, 2010; Alber \& Tièche Christinat, 2011).

En s'inscrivant dans ce numéro thématique de la RSSE, notre proposition montre un exemple des pratiques enseignantes de l'entrée dans l'écrit. Notre intérêt pour la population d'enfants sourds nous dirige vers les recherches qui les concernent, et mentionnent, décrivent ou analysent ces pratiques. Très peu de ces recherches sont menées directement en classe et en situation d'enseignement/apprentissage (p.ex. Bouvet, 1982; Ewoldt, 1990; Krausneker, 2003; Mugnier, 2006a). Il manque également des recherches adoptant une approche compréhensive/interprétative, s'inscrivant dans une recherche qualitative (Mayer, 2007). Or, les pratiques éducatives/enseignantes et leur analyse constituent une clé d'ouverture essentielle dans le but d'améliorer l'éducation de ces enfants et de leur offrir un cadre adapté à leurs besoins spécifiques. Leurs résultats scolaires en dépendent, leur permettant un choix professionnel plus large et un épanouissement social (Bertin, 2007; Courtin, 2007; Hauser \& Marschark, 2008; Mugnier, 2006c). Dans le contexte scolaire, l'amélioration des résultats en lecture/écriture des enfants sourds s'avère primordiale.

Dans la suite de cette contribution, nous proposons de décrire brièvement les approches bilingues dans l'éducation des enfants sourds (point 2). Ensuite, nous ferons une incursion, non exhaustive sur les études en littéracie émergente en général, en nous attardant plus particulièrement sur l'apport de microgenèses didactiques, pour passer ensuite aux études en littéracie émergente menées 
auprès des enfants sourds (point 3). Ceci nous permettra d'entrer dans la partie expérimentale (point 4) qui comprend l'analyse a priori de l'album étudié en Lecture Interactive pour définir les composantes potentielles du savoir littéracique. Ces composantes sont ensuite comparées avec un extrait de protocole d'analyse des interactions en classe pour illustrer leur apparition in vivo, dans le déroulement même de l'activité. Pour conclure (point 5), nous mentionnons quelques perspectives ouvertes par notre travail, en particulier pour la recherche, mais également pour l'établissement de pratiques littéraciques bilingues pour les enfants sourds.

\section{Cadre bilingue en éducation des enfants sourds et ses principes}

\section{Reconnaissance des langues de signes comme moteur du bilinguisme}

Les programmes bilingues sont intimement liés à la reconnaissance législative, linguistique et sociale de la langue des signes (ci-après LS) dans un pays, région, ou société donnée (pour l'Europe, voir Leeson, 2006). Cette reconnaissance suit toutefois les influences historiques et idéologiques, les tensions politiques, les continuités et les ruptures qui marquent l'éducation proposée aux enfants sourds. Nous donnons ici un bref rappel historique. La première école bilingue où la LS a été reconnue et enseignée en tant que langue d'enseignement des savoirs scolaires, est l'école initiée par l'Abbé de l'Epée à partir de 1760 (fondée officiellement comme l'Institut Saint-Jacques en 1794). Cette approche bilingue marque la reconnaissance de la LS dans le monde et initie ce type d'enseignement proposé aux enfants sourds, qui se propage en Europe à l'époque des Lumières. Malheureusement, au siècle suivant, l'interdiction d'utilisation des signes dans l'éducation des enfants sourds dictée par le Congrès de Milan en 1880 marque une rupture dans l'éducation des sourds. Cette rupture est lourde de conséquences car, pendant 100 ans, l'éducation de ces enfants va de pair avec un déni des LS et reste majoritairement ou exclusivement oraliste (en Europe). Un tournant nouveau est marqué par l'apparition en 1960, d'un travail pionnier d'un linguiste américain, William Stokoe, sur la langue des signes américaine (ciaprès ASL). Cette description linguistique de l'ASL provoque une vague de revendications des Sourds (dans les années 1970) à leur langue, à leur culture et à l'éducation de leurs enfants en LS, considérée comme leur langue première. Ces revendications arrivent de l'autre côté de l'Atlantique dans les années 1980 avec une reconnaissance des LS dans les pays européens variable encore aujourd'hui (Leeson, 2006). Cette revendication mène aussi aux débuts des études sur les langues des signes. Avec cette nouvelle reconnaissance on revient aux modèles bilingues d'enseignement aux enfants sourds qui se propagent selon les deux versions (voir plus loin). Entre ces va-et-vient de l'histoire, entre la reconnaissance 
et la fascination pour les LS ou leur déni total, la scolarité des enfants sourds doit trouver sa place dans le monde en évolution sociale et économique constante.

\section{Écoles ou programmes bilingues pour enfants sourds}

Les programmes bilingues, appelés aussi $\mathrm{Bi}-\mathrm{Bi}$ (pour Bilinguisme et Biculturalisme), sont élaborés selon les cinq principes (Niederberger, 2005, p.350): 1/ les LS et la culture des Sourds doivent être considérées de manière équivalente à celles de la société environnante, $2 /$ les enfants sourds tirent des bénéfices de la maîtrise des deux langues, 3/ l'acquisition de la LS ne subit pas d'entraves de la déficience et elle est la langue première de ces enfants, 4/ la LS est utilisée pour l'enseignement des autres matières y compris pour l'enseignement de la langue nationale (ou régionale), $5 /$ cette dernière, qui ne peut pas être apprise naturellement, est une langue seconde pour l'enfant et elle doit lui être enseignée essentiellement dans sa forme écrite, plus accessible. Ces principes se déclinent en programmes des deux types (Niederberger, 2005, p. 351):

Le bilinguisme du type A privilégie la LS comme langue première et celle des apprentissages (Suède et plusieurs écoles aux USA). Dans ces programmes la langue seconde, nationale, est proposée dans un deuxième temps et uniquement dans sa version écrite. Le bilinguisme du type B (introduit dans certaines écoles aux USA et dans plusieurs pays en Europe, dont la France et la Suisse romande) propose une exposition le plus tôt possible aux deux langues, et la langue nationale est introduite dans ses deux versions orale/écrite.

Toutefois, en France, la reconnaissance officielle de la langue des signes française (ou LSF) ne change pas la situation des établissements scolaires pour enfants sourds. La situation est largement insatisfaisante (Bertin, 2007; Dalle, 2003; Mugnier, 2006a, b) et marquée par les inégalités. Mugnier (2006a, b) souligne que, dans la plupart des écoles accueillant ces élèves, il ne s'agit pas d'un bilinguisme réel reconnaissant la valeur et l'importance des deux langues mais d'un bi-monolinguisme, où les deux langues sont séparées et enseignées de manière inégale (p. ex. nombre d'heures).

En Suisse, bien que la Confédération ne reconnaisse pas officiellement les LS, elles sont souvent reconnues par les cantons ou écoles en utilisant la loi de «liberté linguistique» (Hadorn, 2008). A Genève, le programme bilingue au Centre pour enfants sourds de Montbrillant (CESM), est introduit en 1980/81 et il se défend parmi les autres possibilités de prises en charge éducatives. Il propose un enseignement bilingue simultané des deux langues. Les enseignants travaillent en binôme, enseignant sourd/enseignant entendant, et proposent le contact permanent avec les deux langues et dans plusieurs activités. 
Vers une meilleure compréhension de ce bi-plurilinguisme La notion de bilinguisme peut être tout d'abord comprise comme une notion sociale ou comme une notion individuelle (Mugnier, 2006a, pp. 44-57). Nous suivrons dans les grandes lignes cette distinction. En effet, nous pouvons pointer, d'une part, le plan de la société qui utilise deux ou plusieurs langues en tant que langues officielles, comme c'est le cas de la Confédération, avec ses questions de politique linguistique et la reconnaissance des langues des minorités. Ce plan nous intéresse ici au niveau institutionnel et à celui d'une institution particulière, celle de l'école. D'autre part, nous nous intéressons au bilinguisme individuel, celui des utilisateurs de deux ou plusieurs langues, qui nous dirigent vers les travaux phares sur le parler bilingue et ses aspects non seulement discursifs, mais aussi cognitifs ou concernant les apprentissages (Castellotti, 2001; Coste, 1994; Gajo \& Mondada, 2000; Lüdi \& Py, 2003 parmi d'autres). Nous relevons de ces travaux la notion du répertoire verbal (pour nous du répertoire verbal/signé) qui permet d'envisager la compétence bilingue comme la connaissance spécifique des deux langues, qui differe des connaissances langagières des locuteurs monolingues. Cette compétence s'observe chez les locuteurs bilingues même s'ils maitrisent les deux langues seulement d'une manière partielle. Elle est renforcée par la capacité de passer de l'une à l'autre langue selon le besoin et les contacts des langues au fil de la vie (Grosjean, 2004; Moore, 2006; Mugnier, 2006b).

Quant aux enfants sourds, leur bilinguisme particulier, souvent partiel, peut être vu de la même manière, avec une approche de développement d'un répertoire bilingue verbal/signé (Grosjean, 2004). À notre sens, au début de la vie et de la scolarité, cette approche ouvre une perspective intéressante pour ces enfants. Elle permet le développement des connaissances du monde environnant à travers l'une ou l'autre langue. Cette construction des connaissances et du répertoire bilingue en même temps, constitue le défi de la prise en charge précoce de ces enfants. L'entrée dans la communication aisée avec son environnement et le développement du langage (verbal/signé) est particulièrement important dans l'approche des savoirs scolaires, et parmi eux, dans l'apprentissage de la lecture et de l'écriture.

\section{L'importance de L'apprentissage de la lecture/écriture pour jeunes sourds}

Les résultats en lecture/écriture chez les sourds sont accablants en comparaison de ceux des enfants entendants de leur âge. Les jeunes sourds ne dépassent souvent pas le niveau de $3^{\mathrm{e}}$ primaire (Allen, 1986; Traxler, 2000; cités par Marschark et al., 2009, p. 357). On le sait, cet apprentissage est d'une importance capitale dans la vie sociale, en grande partie basée sur la compréhension des messages écrits. Pour ceux qui sont touchés par la déficience auditive, l'entrée dans la communication et dans le langage est difficile, l'élaboration de la langue parlée longue et laborieuse, l'entrée dans le monde de l'écrit présente de nombreuses difficultés (pour un aperçu Lepod-Froment \& Clerebaut, 1996). 
C'est pourquoi l'utilisation de la LS peut constituer une voie d'ouverture, car son apprentissage et son utilisation ne sont pas contraintes par la déficience (Chamberlain, Morford \& Mayberry, 2000; Niederberger, 2007; Padden \& Clark, 2005; Padden \& Ramsey, 1998). Cependant, cette possibilité est loin d'être une solution miracle qui résout toutes les difficultés. Comme le souligne à juste titre Mugnier (2010, p.1), la question de la LS pour les enfants sourds en tant que la langue "maternelle» se pose dans le cas majoritaire où ces enfants naissent dans les familles entendantes ( $90 \%$ de cette population d'enfants). Les parents ne sont donc pas des locuteurs «natifs» de la LS. En conséquence son apprentissage est souvent limité et se déroule à l'école. Cette situation particulière bouleverse notre compréhension du terme «langue maternelle», parce que d'un côté l'enfant n'a pas l'accès à la langue familiale qui est vocale, et de l'autre la famille ne maîtrise pas, en principe, la langue potentiellement accessible à l'enfant, la LS, spatiale et gestuelle.

\section{Études en Littéracie émergente et les apports de microgenèses didactiques}

\section{Littéracie émergente - un courant de recherche et une pratique enseignante}

La littéracie émergente apparaît dans les années '70 pour s'opposer au courant de «reading readiness» qui proclamait la nécessité d'attendre que les enfants soient prêts, assez mûrs pour entrer dans le monde de l'écrit (Schirmer \& Williams, 2003, pp. 110-111). Ce courant contestataire marque l'éducation et les pratiques éducatives adressées aux jeunes enfants avant leur entrée dans les apprentissages formels de la lecture/écriture. Ainsi, ces dernières années, la recherche se développe dans les deux lieux privilégiés où ces pratiques s'exercent: dans les institutions prenant en charge les enfants d'âge préscolaire et dans les familles (voir les autres contributions de ce volume). Nous assistons alors à une multitude de recherches qui décrivent, analysent et évaluent ces pratiques dans les deux cadres. D'importantes études sont menées sur l'efficacité de ces pratiques et leur influence sur la réussite scolaire ultérieure des enfants, en particulier ceux qui peuvent être en difficulté (p. ex. Morin \& Montésinos-Gelet, 2007).

\section{Apports des microgenèses didactiques}

Les microgenèses didactiques se placent parmi les autres courants d'études dans le champ de la littéracie émergente (Saada-Robert \& Balslev, 2006), lorsqu'elles étudient les pratiques littéraciques dans leur déroulement même, en classe. Les situations didactiques proposées par une démarche de recherche sur l'entrée dans l'écrit, peuvent être considérées comme un moyen d'enseignement, construit en collaboration entre les enseignants et les chercheurs (Saada-Robert et al., 2003). Les pratiques littéraciques y sont comprises comme les activités complexes parmi 
lesquelles la Lecture Interactive prend une place importante (Balslev et al., 2005).

Les microgenèses didactiques nous servent également comme l'outil d'analyse compréhensive et interprétative, inductive et déductive à la fois (Balslev \& SaadaRobert, 2007). Elles visent à appréhender les processus d'enseignement/ apprentissage en tant qu'une coconstruction des significations et des connaissances dans une zone de compréhension commune (Balslev, 2006). C'est à travers cette zone que les partenaires du même processus (enseignants et élèves) se retrouvent afin de transposer/saisir les savoirs à enseigner/apprendre. Ces transformations se font par l'intermédiaire des significations que les uns et les autres donnent aux objets d'enseignement et d'apprentissage. Précisément, c'est au cours de l'interaction didactique, pendant la séance, que leurs négociations prennent place dans cette zone intersubjective de construction du sens (Saada-Robert \& Balslev, 2004), considérée aussi comme une zone d'ajustements réciproques (Gamba, 2006).

Recherches en littéracie émergente auprès des enfants sourds Le résumé des recherches sur les pratiques littéraciques auprès des enfants sourds (Schirmer \& Williams, 2003, pp. 112-113) décrit plusieurs activités proposées à ces enfants dans différents types d'écoles. Par exemple, Andrews et Mason (1991) parlent d'une série "d'interventions" en lecture autour des albums et la reconnaissance des mots auprès des jeunes enfants sourds fréquentant l'école résidentielle pour sourds (bilingue du type A). Tandis que Rowe et Allen (1995) décrivent les pratiques littéraciques dans une école publique, où les deux enseignants (sourd et entendant) explorent avec leurs élèves (de 5 à 8 ans) des albums de jeunesse en deux langues. Quant à Bilson, Hayes et Luetke-Stahlman (1996), ils étudient les pratiques littéraciques des enseignants par entretiens (21 écoles, 57 entretiens), en posant leurs questions sur comment et combien ces derniers lisent aux enfants, quels livres ils choisissent etc. Les résultats de cette étude montrent que les enseignants utilisent en majorité la manière explicite d'entrée dans la lecture d'albums, abordent souvent le vocabulaire avant l'activité ou donnent des explications pendant la durée de celle-ci. La majorité utilise soit la LS, soit un code visuel accompagnant la parole en classe.

Quant aux pratiques littéraciques familiales, Swanwick et Watson (2007) les explorent auprès des familles entendantes et sourdes (des enfants sourds). Ils montrent une différence importante entre ces deux groupes de parents. Les parents sourds engagent leur enfant dans un discours en LS sur la base des images, favorisent les hypothèses émises par l'enfant. Pour, leur part, les parents entendants lisent le texte en pointant les mots et en attirant ainsi l'attention de leur enfant sur ces aspects lexicaux en lien avec les images du livre.

Une revue de littérature concernant la littéracie émergente (Williams, 2004) mentionne l'existence d'environ 20 recherches spécifiques sur les pratiques littéraciques conduites auprès des enfants sourds. Cependant, ces recherches sont souvent des études de cas qui ne suffisent pas pour améliorer ou introduire des changements plus généraux dans l'éducation de ces enfants. 


\section{Lecture Interactive comme exemple d'une pratique Littéracique complexe}

\section{Participants et terrain d'observations}

Notre contribution émane d'une recherche exploratoire, préalable à une étude doctorale plus large. Les deux recherches ont été menées dans une classe bilingue LSF/français, spécialisée dans l'accueil des enfants sourds et intégrée dans une école publique de la ville de Genève. La recherche exploratoire (en deuxième enfantine) constitue une première approche du terrain d'observations. Elle s'intéresse plus particulièrement aux pratiques enseignantes réellement mises en jeu lors des séances de travail autour de l'album de jeunesse. Comment les enseignants et les élèves interagissent-ils et se comprennent-ils? Comment les enseignants mènent-ils la leçon ? Comment appréhendent-ils l'album et les significations qui en émergent? Comment les deux langues participent-elles dans cette construction conjointe des savoirs littéraciques?

Analyse a priori de L'album "La maîtresse fait un vol plané» L'album lu en classe ainsi que le dispositif didactique qui l'accompagne servent aux enseignants d'outil de transposition du savoir lire/écrire. Nous nous intéressons au texte de l'album de littérature enfantine pour relever ses caractéristiques de genre, et souligner ses propriétés potentielles (Adam, 1992). La richesse de cet album apparaît tout d'abord dans les caractéristiques du texte. Il s'agit d'un récit vraisemblable, proche des pratiques de la vie scolaire réelle, narré par un garçon, Rémi, d'environ 5-6 ans. Sa structure narrative est complexe et son action se déroule en un laps de temps d'une semaine. La vie quotidienne de la classe est perturbée par l'accident de la maîtresse et ses conséquences. Son épilogue a lieu deux mois plus tard lorsque la maîtresse revient définitivement en classe. Les événements pris en compte par cette structure basée sur la vie scolaire sont complétés par d'autres éléments de la vie quotidienne du narrateur qui se déroulent dans son cadre familial. Le texte est généralement soutenu par l'image, et pourtant cette adéquation n'est pas respectée dans tous les cas. Certains éléments d'illustration servent soit de jeu pour mieux stimuler l'observation des enfants, lecteurs potentiels, soit d'éléments complémentaires aux traits psychologiques qu'on ressent et que le texte ne dit pas forcément, comme le cadre familial de Rémi, chaleureux et calme souligné par les couleurs pastels etc. Les composantes thématique, sémantique et syntaxique du texte sont appropriées pour les enfants de l'âge concerné.

\section{Dispositif de Lecture Interactive et savoir à enseigner}

Les prescriptions du programme scolaire de l'école genevoise pour le premier cycle d'apprentissages sont définies en termes de préparation, d'initiation au monde de l'écrit et de développement d'une aptitude adaptée aux futurs apprentissages (DEP, 2000). Comment ces prescriptions se déclinent-elles face à la 
lecture/écriture? L'apprentissage de la littéracie est englobé par les objectifs concernant l'apprentissage de la langue. Ceux-ci comprennent d'une part l'observation du fonctionnement de la langue (ses structures et régularités, ses particularités) et d'autre part la production et la compréhension de textes, de genres différents, à l'oral et à l'écrit. Une attention particulière est consacrée à l'initiation au graphisme et à l'acte d'écrire. Ces objectifs généraux sont pris en compte et adaptés selon les besoins spécifiques des enfants déficients auditifs par l'équipe des enseignants et des collaborateurs (sourds et entendants) d'une institution spécialisée (CESM), dépendante de l'Office médico-pédagogique (OMP) faisant partie du Département de l'Instruction Publique (DIP).

Les objectifs que les enseignants observés mentionnent pour cette année scolaire peuvent se résumer comme suit: $1 /$ augmenter le vocabulaire des élèves dans les deux langues, $2 /$ les familiariser avec plusieurs genres de textes écrits et $3 /$ les amener à prendre en compte les propriétés linguistiques du texte pour en contrôler la compréhension. La réalisation de ces objectifs passe par plusieurs activités complexes (comme lire ensemble l'album) ou par des activités spécifiques centrées sur les tâches plus précises, par exemple: la reconnaissance globale de mots accompagnés d'images, l'étude des structures sublexicales comme l'identification et la production des lettres. Elle est plus facile et plus rapide à apprendre en LSF par l'appropriation de l'alphabet manuel. Les autres types de tâches peuvent être proposées aux enfants comme l'identification des syllabes, l'initiation à la reconnaissance des quelques sons de la langue française, une tâche très difficile pour ces enfants et travaillée aussi en logopédie de manière individuelle.

La Lecture Interactive de cet album est constituée de cinq phases: une présentation du déroulement et du but de l'activité; l'exploration de la couverture du livre, de son image et de son titre; la lecture interactive avec hypothèses où les enfants explorent l'album page par page, guidés par leurs enseignants; puis la vérification des hypothèses par la lecture complète de l'album en deux langues; une consolidation de la compréhension du récit à travers des activités diverses, proposées ou non par le guide pédagogique et le matériel didactique accompagnant l'album (Fabre \& Fabre, 2000). Dans une séance de travail, ces phases peuvent être mélangées, l'exploration du livre et la lecture avec hypothèses progressent au rythme des enfants et sont, au besoin, entrecoupées par les exercices de consolidation.

\section{Analyse effective de la première séance de Lecture Interactive}

Dans cette analyse, il s'agit de considérer les composantes du savoir littéracique, potentiellement présentes dans l'album, cette fois-ci effectivement enseignées et la dynamique des apprentissages qui se déroulent dans la situation même de 
Lecture Interactive. Autrement dit, nous tentons d'appréhender un objet d'étude triadique de la construction des savoirs tels qu'ils fonctionnent en situation didactique où l'objet d'enseignement/apprentissage évolue pendant l'interaction entre les deux partenaires, les élèves et leurs enseignants.

L'extrait analysé ici est tiré de la première séance de lecture interactive de l'album analysé plus haut. Cette séance a pour but la découverte de la page de couverture, l'exploration de son image d'abord partiellement avec le cache à quatre fenêtres proposé par le guide pédagogique. Ensuite, elle prévoit le travail sur la couverture entière et sur le texte qui s'y trouve (le titre et les noms des auteurs).

À partir d'un enregistrement vidéo, l'ensemble de la séance a été transcrit en protocole complet et continu. Le tableau 1 présente le troisième niveau des procédés d'analyse microgénétique (Balslev \& Saada-Robert, 2007; Gamba, 2006) qui consiste à interpréter/catégoriser les unités de sens préalablement définies à partir des tours de paroles. Ces unités (ci-après US) sont comprises comme les plus petites unités d'analyse auxquels les partenaires attribuent du sens en relation avec les contenus de savoir. L'enchaînement de plusieurs US relevant du même contenu constitue une séquence microgénétique. La première séquence de ce protocole représente la mise en marche de l'activité qui dirige l'attention des enfants vers l'album exposé sur la table. Pour découvrir progressivement le contenu de la page de couverture, les enseignants ont prévu un cache avec des fenêtres ouvertes dans un ordre précis. L'enseignant entendant (EE) explique le déroulement de l'activité: "On va réfléchir, on va découvrir le livre, on va découvrir la couverture, ensemble on va voir, je vais ouvrir ça (EE pointe les fenêtres) et on va regarder et réfléchir, on voit quoi, c'est quoi, ça veut dire quoi». Ces explications sont entrecoupées par les interventions des enfants et enfin l'activité démarre par la deuxième séquence qui commence non pas par la communication verbale/signée, mais par l'action d'EE qui ouvre la première fenêtre sur la page de couverture (tableau 1). 
Tableau 1. Extrait du protocole organisé de l'album "La maîtresse fait un vol plané», première séance.

\begin{tabular}{|c|c|c|c|}
\hline US & $\begin{array}{l}\text { Enseignante entendante - EE } \\
\text { Enseignant sourd - ES }\end{array}$ & Elèves - Igo, Ade, Geo, Bar & $\begin{array}{l}\text { Contenu du } \\
\text { savoir }\end{array}$ \\
\hline \multicolumn{4}{|c|}{ Séquence II: repérage de l'héroïne sur la base de l'image } \\
\hline 28c) & $\begin{array}{l}\text { EE: /alors (elle ouvre la première fenêtre } \\
\text { du cache-couverture) }\end{array}$ & & PROGACT \\
\hline 29a) & & I: maman MAMAN/ & PICFORM \\
\hline $29 b)$ & & (à A) VOILÀ & PICFORM \\
\hline 30 & $\begin{array}{l}\text { ES: (à I) TOI MAMAN PEUT-ÊTRE } \\
\text { REGARDE }\end{array}$ & & PICFORM \\
\hline 31a) & EE: c'est qui QUI?// & & PICFORM \\
\hline 31b) & lui il dit maman IL DIT MAMAN & & PICFORM \\
\hline 31c) & tu vas voir & & PROGACT \\
\hline 31d) & (se lève pour écrire au tableau maman) & & LEX \\
\hline 32 & & A: moi aussi AUSSI & PICFORM \\
\hline 33a) & $\begin{array}{l}\text { EE: (à A) toi aussi tu dis c'est maman } \\
\text { TOI TU DIS MAMAN }\end{array}$ & & PICFORM \\
\hline $33 \mathrm{~b})$ & (à G) toi aussi? TOI/ & & PICFORM \\
\hline 33c) & toi tu dis c'est qui? TOI TU DIS QUI & & $\begin{array}{l}\text { PICFORM } \\
\text { PROGACT }\end{array}$ \\
\hline 34 & & G: maman MAMAN & PICFORM \\
\hline 35 & & A: maman & PICFORM \\
\hline 36 & & B: maman MAMAN & PICFORM \\
\hline 37 & & A: moi j'dis papa & PICFORM \\
\hline 38 & EE: c'est un monsieur ou une dame? & & PICFORM \\
\hline 39 & & A: madame pas monsieur & PICFORM \\
\hline 40a) & $\begin{array}{l}\text { EE: dame donc c'est une maman } \\
\text { MAMAN/ }\end{array}$ & & PICFORM \\
\hline 40b) & $\begin{array}{l}\text { c'est pas possible PAS POSSIBLE papa } \\
\text { PAPA }\end{array}$ & & PICFORM \\
\hline 41 & & A: elle est chou la dame & PICFORM \\
\hline \multicolumn{4}{|c|}{ Séquence III: boucle lexicale-sémantique : apprentissage d'un nouveau signe } \\
\hline 42 & ES: UNE DAME UNE DAME & & LEXSEM \\
\hline 43a) & $\begin{array}{l}\text { EE: c'est peut-être PEUT-ÊTRE } \\
\text { maman/on sait pas SAIT PAS }\end{array}$ & & PICFORM \\
\hline 43b) & et ES il dit UNE DAME & & LEXSEM \\
\hline 43c) & (écrit au tableau une dame) & & LEX \\
\hline 44 & $\begin{array}{l}\text { ES: UNE DAME EN LANGUES DES } \\
\text { SIGNES UNE DAME }\end{array}$ & & LEXSEM \\
\hline 45 & & I: UNE DAME UNE DAME BRAVO & LEXSEM \\
\hline 46a) & $\begin{array}{lll}\text { EE: on peut signer } & \text { POUVOIR } \\
\text { SIGNER UNE DAME } & \end{array}$ & & LEXSEM \\
\hline 46b) & regardez/regardez (pointe ES) & & $\begin{array}{l}\text { LEXSEM } \\
\text { ENGA+ }\end{array}$ \\
\hline 47 & ES: MADAME OU UNE DAME & & LEXSEM \\
\hline 48 & & G: UNE DAME UNE DAME & LEXSEM \\
\hline 49 & & B: ah ES ! ES ! ES ! UNE DAME & LEXSEM \\
\hline 50 & EE: (à A) une dame UNE DAME & & LEXSEM \\
\hline
\end{tabular}




\begin{tabular}{|l|l|l|l|}
\hline 51 & & A: UNE DAME & LEXSEM \\
\hline 52 & ES : (à B) BIEN & & LEXSEM \\
\hline $53 \mathrm{a})$ & EE : (à B) BIEN bien & & LEXSEM \\
\hline $53 \mathrm{~b})$ & (à ES) alors lui PROGRESSE BIEN & & ENGA+ \\
\hline 54 & \multicolumn{2}{|l|}{ G: UNE DAME } & LEXSEM \\
\hline Séquence IV : composante sémantique & $\begin{array}{l}\text { PICFORM, } \\
\text { PROGACT }\end{array}$ \\
\hline 55 & EE: et puis elle fait quoi ? QUOI FAIRE & &
\end{tabular}

Légende:

En MAJUSCULES - les signes en LSF; en minuscules - les énoncées dits; dans le cas des enfants ou de EE plusieurs énoncées dits sont répétés en langue des signes ou signés en même temps.

PICFORM - l'énoncé porte sur la description de l'image, prise isolément du récit complet, du livre, ou sur ses aspects esthétiques, formels;

LEX ou LEXSEM- l'énoncé porte sur le repérage d'un mot et/ou sur le sens à en tirer;

PROGACT- lorsque l'énoncé sert à faire progresser ou à organiser l'activité;

ENGA+ - l'énoncé porte sur la posture d'engagement ou de désinvestissement par rapport au savoir.

On peut voir dans le tableau 1 une évolution des composantes du savoir, d'une séquence d'échanges sur le contenu de l'image (PICFORM) vers une séquence sur le lexique (LEXSEM), et ensuite le retour vers l'exploration de l'image.

Comment se co-construit une zone de compréhension commune entre les partenaires?

L'extrait ci-dessus contient des éléments saillants d'interactions enseignantsapprenants qui nous permettent d'observer les processus fins de construction conjointe d'une zone de compréhension. Le premier élément relève d'un échange typique pour la LSF qui vise l'apprentissage d'un signe nouveau (ici UNE DAME, désignant une personne qu'ils voient sur l'image et qui représente l'héroïne du récit). L'enseignant sourd (ES) interrompt le déroulement de la séquence à dominance sémiopicturale centrée sur l'image (PICFORM) pour donner une autre possibilité d'expression aux enfants. Cette interruption centrée sur le contenu du savoir lexical, mais aussi sémantique, porte sur la signification du signe proposé et mieux approprié au besoin de la situation. Ensuite, plusieurs répétitions ont pour but l'apprentissage correct du signe et de ses caractéristiques structurelles, telles que son emplacement, sa configuration, son mouvement, etc. Cet échange répond à une demande implicite de la part d'Ade qui essaie de donner une autre proposition que «maman» pour l'image qu'elle voit (US 3741). Cette partie du protocole montre une sensibilité des enseignants à exploiter toutes les propositions des enfants qui explorent le livre et qui, par ce fait, apprennent une grande partie de leur vocabulaire qui fondera par la suite leur répertoire actif bilingue (des mots/signes) à utiliser dans les autres situations, mais aussi des concepts qui les accompagneront et qui constitueront leurs connaissances du monde. 
Le deuxième élément est formé par les US 31d et 43c, relevant d'une composante de savoir lexical, il s'agit ici de l'écriture au tableau noir de deux mots: maman et une dame. Cet élément est une amorce du travail qui sera fait ultérieurement, et qui consiste à comparer ces deux mots écrits au tableau avec le mot maîtresse appartenant au titre de l'album, que les enfants découvrent dans la deuxième fenêtre du cache couverture. Ces deux exemples montrent déjà la richesse des échanges entre les partenaires, ainsi que la complexité dans laquelle ils se trouvent. Par ailleurs, les enseignants et les apprenants font preuve d'ouverture et d'une grande capacité d'écoute l'un de l'autre, et d'observation, pour entrer en dialogue. D'un côté, les enseignants proposent et rendent possible l'apparition d'une zone de compréhension commune et de l'autre, les élèves l'acceptent en la co-construisant. Elle apparaît autour des composantes du savoir comme une zone de rencontre (Grossen \& Py, 1997) dans laquelle les uns et les autres peuvent élaborer des significations partageables, évoluant jusqu'à ce qu'elles deviennent partagées ou considérées comme telles (Balslev, 2006; SaadaRobert \& Balslev, 2004).

Hormis ce court extrait comment se passe la suite de cette séance? Comment les enseignants mettent-ils en évidence plusieurs supports? Comment les exploitent-ils? Quels types de tâches proposent-ils aux enfants? Quels échanges sont ainsi engendrés et autour de quelles composantes du savoir lire/écrire?

Après avoir ouvert toutes les fenêtres du cache, les enfants guidés par leurs enseignants explorent la première page de couverture en entier, en mentionnant les éléments de l'image et en émettant leurs hypothèses quant au contenu du récit. Puis, ils sont invités par les enseignants à regarder le titre et à compter les mots qui le composent. Les enseignants leur proposent ensuite de mettre en ordre ces mots dans une tâche d'écriture sur une fiche séparée. Finalement, ils reviennent à la lecture du livre et lisent en deux langues le texte de deux pages, qui présente les personnages de l'histoire et le contexte de la situation initiale. Dans tous ces moments distincts, les deux langues leur servent d'intermédiaire et de support. Les enseignants utilisent souvent le tableau pour y écrire les mots qui sont ensuite pointés, soulignés, comparés, utilisés dans la négociation des significations émergentes.

\section{Conclusions et perspectives}

Comme le soulignent les chercheurs de pointe dans ce champ (Hauser \& Marschark, 2008), il manque une réelle réflexion concernant les pratiques enseignantes et éducatives dans plusieurs disciplines scolaires et dans tous les types de classes qui accueillent les enfants sourds. Notre contribution tente d'y apporter une bribe, en montrant une recherche concernant les jeunes enfants sourds observés en classe lors de la lecture partagée, interactive de l'album de jeunesse.

Les conclusions de notre recherche exploratoire ont permis la mise en place d'une recherche doctorale plus conséquente (Tominska, 2005). Ces observations 
ont été primordiales pour rendre compte des pratiques littéraciques bilingues, riches et diversifiées, qui favorisent l'accès des élèves aux contenus du savoir lire/écrire présents dans cette activité. Ces contenus sont l'objet d'une coconstruction en interaction grâce à l'intermédiaire des deux langues. Les enfants commencent à les maîtriser de manière non équivalente, mais progressive. De cette manière, l'une peut servir à l'étude et au développement de l'autre. Le rôle de la LS dans la construction des significations tirées des images et progressivement du texte, est non négligeable. Les médiations de l'enseignant sourd en témoignent. Elles sont importantes dans la compréhension de l'activité de manière globale, dans l'élaboration des hypothèses à partir de l'image et du texte, dans la négociation des significations apparaissant durant l'échange. Ces médiations interviennent aussi dans la construction du lexique émergeant de l'album ainsi que dans l'étude des unités linguistiques telles que les lettres. Les enfants se montrent en tant que partenaires à part entière, sont actifs et s'engagent dans ce type d'échange bilingue et multimodal par excellence (de Saint-Georges, 2008). Leurs réponses/interventions sont accueillies dans les deux langues, en permettant les mélanges, les passages de l'une à l'autre langue selon les connaissances construites par chaque enfant. Ainsi, les imperfections sont corrigées dans les deux langues, par l'un ou l'autre enseignant en charge de la classe. Il s'agit bien là d'un parler bilingue, plein de flux et reflux entre ces deux langues présentes en classe (Grosjean, 2004). La force de cette démarche bilingue réside dans la reconnaissance et l'utilisation permanente des deux langues, dans un but commun de construction des connaissances langagières/littéraciques des enfants, afin d'ouvrir vers les autres apprentissages scolaires.

\section{Vers des nouvelles recherches}

Les chercheurs en surdité (Hauser \& Marschark, 2008, et autres) soulignent la nécessité de nouvelles investigations en collaboration étroite avec les enseignants expérimentés et par la suite, de la prise en compte de leurs résultats sur le terrain de l'action pédagogique. Le besoin donc de réformes sensées, soutenues par la recherche, et non pas par des a priori et des présuppositions, est immense. C'est également le but de l'Observatoire de la surdité de préparer la prise des décisions avant que les réformes prennent place (Tièche Christinat \& CSPS, 2010), en connaissance de cause et après une réflexion approfondie, fondée sur les résultats des investigations du terrain.

Une autre nécessité consiste à explorer finement les pratiques enseignantes (en général) dans les classes qui accueillent les enfants sourds. En même temps, une observation détaillée des procédures d'apprentissage et des mesures sur les acquis d'élèves sont nécessaires pour compléter nos connaissances sur les processus fins qui caractérisent l'enseignement/apprentissage en situations didactiques. Comment l'analyse de ces processus peut-elle contribuer à ce qu'enseignants et élèves arrivent au but commun de réussite scolaire et de développement harmonieux dans le monde moderne? La question reste ouverte, il n'y a 
pas une pratique exclusive et l'heure est au dépassement des vieilles querelles (Courtin, 2007; Marschark, 2007). Le but est de concevoir des pratiques et des programmes efficaces pour un réel changement dans la prise en charge de ces enfants.

Ces derniers, constituent un groupe hétérogène avec des sous-groupes, par exemple les enfants implantés de plus en plus nombreux, dont les besoins en matière d'éducation ne sont pas encore assez connus ni assez étudiés. Les constats de l'Observatoire vont dans le même sens indiquant cette nouvelle population d'enfants sourds comme distincte et à besoins spécifiques en matière de scolarité (Tièche Christinat \& CSPS, 2010).

Les pratiques littéraciques pour les enfants sourds nécessitent, elles aussi, plus d'investigations scrupuleuses, afin d'examiner plus finement comment certains enfants ont réussi à devenir de très bons lecteurs. Quelles étaient leurs trajectoires d'apprentissage et les facteurs qui ont favorisé leur réussite et leur haut niveau de connaissances littéraciques? Comment ont-ils fait pour dépasser l'apparent blocage de passage du niveau du lecteur débutant au niveau du lecteur autonome et fluide (Mayer, 2007)?

\section{Vers des pratiques littéraciques bilingues pour enfants sourds}

Nous avons présenté un exemple de pratiques littéraciques bilingues observées en classe pour enfants sourds à Genève. Les résultats de notre recherche doctorale montrent que les élèves profitent de ces pratiques littéraciques bilingues où les deux langues favorisent la compréhension des objets d'enseignement/apprentissage et la construction de connaissances littéraciques des élèves. Dans le cas de cette recherche longitudinale, cette construction est observable dans l'évolution des élèves aux bilans psycholinguistiques du début à la fin de l'année scolaire. Nous montrons aussi que les enfants élaborent et enrichissent leurs répertoires bilingues et profilent des dominances selon leur choix de langue. Ces résultats corroborent d'autres recherches qui proclament l'introduction de vraies pratiques bilingues éducatives et scolaires pour enfants sourds (Bertin, 2007; Millet et al., 2009; Mugnier, 2006c, 2010). Et surtout, dans le monde de mobilité et d'échanges linguistiques d'aujourd'hui, le bilinguisme sourd ne devrait pas être une exception, mais au contraire, il devrait être compris et accepté en tant qu'une richesse, une ressource, un moteur de développement. Exactement comme pour d'autres enfants qui vivent les situations de contact des langues et des cultures au quotidien. Dans le débat national sur l'implantation du bilinguisme dans l'école suisse d'aujourd'hui, ce bilinguisme devrait aussi trouver sa place. 


\section{References}

Adam, J. M. (1992). Les textes, types et prototypes: récit, description, argumentation, explication et dialogue. Paris: Nathan.

Alber, S. \& Tièche Christinat, C. (2011). Exposition à la langue orale et scolarisation des enfants présentant un déficit auditif: Extraits des données de l'Observatoire de la surdité en Suisse romande et au Tessin. [Version électronique.] Newsletter CSPS, janvier 2011.

Balslev, K. (2006). Microgenèses didactiques dans une situation de révision textuelle en milieu adulte. Thèse de doctorat en sciences de l'éducation. Faculté de psychologie et des sciences de l'éducation. Université de Genève.

Balslev, K., Claret-Girard, V., Mazurczak, K., Saada-Robert, M. \& Veuthey, C. (2005). La resolution de problems en français scriptural: un outil pour enseigner/apprendre. Revue française de pédagogie, Vol. 150, 59-72.

Balslev, K., Martinet, C. \& Saada-Robert, M. (2006). La lecture interactive d'albums de littérature enfantine à 4 ans en classe. Étude microgénétique. Les Dossiers des Sciences de l'éducation, $\mathrm{N}^{\circ} 15,41-52$.

Balslev, K. \& Saada-Robert, M. (2007). Les microgenèses situées. Unités et procédés d'analyse inductive-déductive. Recherche Qualitative, Vol. 26 (2), 85-109.

Bertin, F. (2007). Les enfants sourds à l'école en France: pour un projet bilingue. [Numéro spécial]. Enfance, 59 (3), 237-244.

Bouvet, D. (1982). La parole de l'enfant sourd. Paris: PUF.

Castellotti, V. (2001). La langue maternelle en classe de langue étrangère. Paris: Clé International.

Chamberlain, C., Morford, J. P. \& Mayberry, R. I. (2000). Language Acquisition by Eye. Mahwah, New Jersey: Lawrence Erlbaum Associates.

Coste, D. (1994). L'enseignement bilingue dans tous ces états. ELA, 96, 9-22.

Courtin, C. (2007). Introduction. L'enfant sourd en développement. Pour une approche globale de son éducation. In C. Courtin (Ed.), Comment peut-on être sourd? [Numéro spécial]. Enfance, 59 (3), 212-219.

Dalle, P. (2003). La place de la langue des signes dans le milieu institutionnel de l'éducation : enjeux, blocages et évolution. In C. Cuxac (Ed.). La langue des signes, statuts linguistiques et institutionnels. [Numéro spécial]. Langue Française, 137, 32-60.

De Saint-Georges, I. (2008). La multimodalité et ses ressources pour l'enseignement-apprentissage. In L. Filliettaz, I. de Saint-Georges \& B. Duc (Ed.). «Vos mains sont intelligentes !». Interactions en formation professionnelle initiale (pp. 117-158). Cahiers de la Section des Sciences de l'éducation. Genève: Université de Genève.

Direction de l'enseignement primaire (DEP). (2000). Les objectifs d'apprentissage de l'école primaire genevoise. Genève: Département de l'instruction publique.

Ewoldt, C. (1990). The Early Literacy Development of Deaf Children. In D. Moores \& K., Meadow-Orlans (Ed.). Educational and Developmental Aspects of Deafness (pp. 85-114). Washington: Gallaudet University Press.

Fabre, D., \& Fabre, E. (2000). Guide pédagogique. ABRACADALIRE. Paris: Hatier.

Gajo, L. \& Mondada, L. (2000). Interactions et acquisitions en contexte: modes d'appropriation de compétences discursives plurilingues par de jeunes immigrés. Fribourg: éditions Universitaires de Fribourg.

Gamba, C. (2006). Démarche méthodologique pour analyser la lecture d'un album sans texte entre une éducatrice et un groupe d'enfants de trois ans. Les Dossiers des Sciences de l'Education, 15, 27-40.

Grosjean, F. (2004). Bilinguisme, Biculturalisme et Surdité. In A. Garouben \& B. Virole (Ed.) Le bilinguisme aujourd'hui et demain. Actes de la journée d'études et de recherches sur la surdité. Paris, novembre, 2003 (pp. 52-70). Paris: CTNERHI \& GERS.

Grossen, M. \& Py, B. (1997). Pratiques sociales et médiations symboliques. Berne: Peter Lang. 
Hadorn, D. (2008, sept.). La langue des signes en Suisse du point de vue juridique. Exposé présenté au 3e Congres de la Fédération Suisse des Sourds (SGB-FSS), intitulé: La langue des signes, un droit pour tous. Locarno, Suisse.

Hauser, P. C. \& Marschark, M. (2008). What we know and what we don't know about cognition and deaf learners. In M. Marschark \& P. C. Hauser (Ed.), Deaf cognition: Foundations and outcomes (pp. 439-457). New York: Oxford University Press.

Krausneker, V. (2003, mai). Deaf Second Language Learning in Elementary School. Report from a Sociolinguistic Research Study in the First Bilingual Class in Vienna, Austria. Paper presented at EDDE- European Days of Deaf Education, University of Örebro, Sweden.

Leeson, L. (2006). Les langues signées dans l'enseignement en Europe - exploration préliminaire. Strasbourg: Conseil de l'Europe.

Lepod-Froment, C. \& Clerebeaut, N. (1996). L'enfant sourd. Communication et langage. Bruxelles: De Boeck.

Lüdi, G. \& Py, B. (1986/2003). Etre bilingue. (3e éd.). Berne: Peter Lang.

Marschark, M. (2007). Comprendre et utiliser les bases cognitives de l'apprentissage chez les enfants sourds. [Numéro spécial]. Enfance, 59 (3), 271-281.

Marschark, M. \& Hauser, P. C. (2008). Cognitive Underpinnings of Learning by Deaf and Hard-of-Hearing Students : Differences, Diversity, and Directions. In M. Marschark \& P. C. Hauser (Ed.), Deaf cognition: Foundations and outcomes (pp. 3-23). New York: Oxford University Press.

Marschark, M., Convertino, C. M. \& LaRock, D. (2006). L'évaluation dans le domaine de la cognition, de la communication et des apprentissages chez les élèves et étudiants sourds. In C. Hage, B. Charlier \& J. Leybaert (Ed.), Compétences cognitives, linguistiques et sociales de l'enfant sourd. Pistes d'évaluation (pp. 26-53). Sprimont: Mardaga.

Marschark, M., Sapere, P., Convertino, C., Mayer, C., Wauters, L. \& Sarchet, T. (2009). Are Deaf Students' Reading Challenges Really about Reading? American Annals of The Deaf. 154(4), 357-370.

Mayer, C. (2007). What Really Matters in the Early Literacy Development of Deaf Children. Journal of Deaf Studies and Deaf Education, 12 (4), 411-431.

Millet, A., Mugnier, S., Sabria, R. \& Simon, D. L. (2009). Surdité et contacts de langue: quelles implications socio-didactiques? In I. Pierozak \& J-M. Eloy (Ed.), Intervenir: appliquer, s'impliquer? (pp. 241-250). Paris: Harmattan.

Moore, D. (2006). Plurilinguismes et école. Paris: Didier.

Morin, M.-F. \& Montésinos-Gelet, I. (2007). Effet d'un programme d'orthographes approchées en maternelle sur les performances ultérieures en lecture et en écriture d'élèves à risque. Revue des Sciences de l'Éducation. Vol. 33 (3), 663-683.

Mugnier, S. (2006a). Surdités, Plurilinguisme et Ecole. Approches sociolinguistiques et sociodidactiques des bilinguismes d'enfants sourds de CE2. Thèse de doctorat. Grenoble: Université Stendhal-Grenoble 3. [version électronique sur: http://tel.archives-ouvertes.fr/tel$00270553 / \mathrm{fr} /]$.

Mugnier, S. (2006b). Le bilinguisme des enfants sourds: de quelques freins aux possibles moteurs. [version éléctronique] GLOTTOPOL, $2006\left(\mathrm{n}^{\circ} 7\right), 144-159$.

Mugnier, S. (2006c). Vers une dynamique bilingue. Étude des interactions français/langue des signes française à l'école primaire. In D. Daigle \& A-M., Parisot (Ed.), Surdité et société. Perspectives psychosociale, didactique et linguistique (pp. 63-79). Québec: Presses de l'Université du Québec.

Mugnier, S. (2010). Mise en lumière des stratégies interactives au sein d'une classe d'enfants sourds. Actes du colloque International "Spécificités et diversité des interactions didactiques: disciplines, finalités, contextes.» UMR ICAR, Université Lyon 2, INRP, CNRS, Lyon, 2426 juin, 2010. [CD-Rom]

Niederberger, N. (2005). Rééducation de l'enfant sourd profond: bilinguisme. In P. 
Dulguerov \& M., Remacle (Ed.), Précis d'audiophonologie et de déglutition. Vol. I: L'oreille et les voies de l'audition, (pp. 349-362). Marseille: Solal.

Niederberger, N. (2007). Apprentissage de la lecture-écriture chez les enfants sourds. [Numéro spécial]. Enfance, 59 (3), 254-262.

Padden, C. \& Clark, D. (2005). Comment l'alphabet devint représenté en langue des signes. In C. Transler, J. Leybaert \& J-E., Gombert (Ed.), L'acquisition du langage par l'enfant sourd. Les signes, l'oral et l'écrit (pp. 71-89). Marseille: Solal.

Padden, C. \& Ramsey, C. (1998). Reading ability in signing deaf children. Topics in Language Disorders, Vol. 18 (4), 30-46.

Saada-Robert, M. \& Balslev, K. (2004). Une microgenèse située des significations et des savoirs. In C. Moro \& R. Rickenmann (Ed.), Situation éducative et significations (pp. 135163). (Raisons éducatives $\mathrm{N}^{\circ} 8$ ). Bruxelles: De Boeck.

Saada-Robert, M. \& Balslev, K. (2006). Les microgenèses situées. Études sur la transformation des connaissances. Revue suisse des sciences de l'éducation, vol. 28 (3), 487-514.

Saada-Robert, M., Auvergne, M., Balslev, K., Claret-Girard, V., Mazurczak, K. \& Veuthey, C. (2003). Ecrire pour lire dès 4 ans. Didactique de l'entrée dans l'écrit. Cahiers de la Section des Sciences de l'Education, $N^{\circ} 100$. Genève: Université, FPSE.

Schirmer, B. R. \& Williams, C. (2003). Approaches to Teaching Reading. In M. Marschark \& P. E. Spencer (Ed.), Oxford Handbook of Deaf Studies, Language and Education (pp. 110122). New York: Oxford University Press.

Schwartz, I. \& Catel (1999). La maîtresse fait un vol plané. ABRACADALIRE. Paris: Hatier.

Swanwick, R., \& Watson, L. (2007). Parents Sharing Books With Young Deaf Children in Spoken English and in BSL: The Common and Diverse Features of Different Language Settings. Journal of Deaf Studies and Deaf Education, 12 (3), 385-405.

Tominska, E. (2005). L'apprentissage de la lecture et de l'écriture chez de jeunes enfants sourds dans le cadre scolaire bilingue LSF/français. Etude microgénétique. Canevas de thèse de doctorat. Genève: Université, FPSE.

Tièche Christinat, C. \& CSPS (2010). Observatoire romand de la surdité. Projet OPERA. Troisième rapport. Lausanne: HEP VD \& CSPS. [version électronique sur : http://www.hepl.ch/uploads/media/rapport_opera_janv_2010.pdf].

Williams, C. (2004). Emergent Literacy of Deaf Children. Journal of Deaf Studies and Deaf Education, 9(4), 352-365.

Mots clés: Pratiques littéraciques, enfants sourds, bilinguisme, lecture interactive, construction du savoir lire/écrire 


\section{Welche literalen Praktiken für gehörlose Kinder?}

\section{Zusammenfassung}

Dieser Artikel beschreibt literale Praktiken in einer zweisprachigen Klasse für gehörlose Kinder. Die Förderkonzepte für gehörlose Kinder werden mit Konzepten ergänzt, die aus der Forschung zu früher Literalität nicht beeinträchtigter Kinder - unter anderem aus Untersuchungen zur Mikrogenese von LehrLernprozessen - stammen. Eine Unterrichtssequenz, in welcher die Lehrperson mit den Kindern interaktiv das Verstehen einer Bilderbuch-Geschichte erarbeitet, veranschaulicht die literalen Fähigkeiten, die in solchen Lehr-Lernarrangements gefördert werden können. In dieser komplexen didaktischen Situation wird deutlich, dass solche Fähigkeiten erst durch die Interaktion evoziert werden, und dass Kinder und Lehrpersonen durch wechselseitige Anpassungen ein gemeinsames Verständnis der Geschichte aushandeln. Untersucht werden auch die Rollen des Mediums und der beiden in dieser Interaktion verwendeten Sprachen.

Schlagworte: Literale Praktiken, literale Förderung, gehörlose Kinder, zweisprachiger Unterricht, interaktives Lesen, Leseverstehen

\section{Quali pratiche di introduzione alla scrittura per i bambini sordi?}

\section{Riassunto}

Questo articolo descrive le pratiche di introduzione alla scrittura introdotte in una classe bilingue (lingua dei segni / francese) per bambini sordi. L'articolo presenta un ancoraggio concettuale dei programmi bilingui per l'educazione di questi bambini, seguito poi da contributi di studi sull'ingresso nella scrittura dei bambini, e tra gli altri, delle microgenesi didattiche. Una sessione di lettura interattiva servirà ad illustrare quali sono le componenti del processo di alfabetizzazione che emergono nel discorso in classe. In questa situazione didattica complessa, cogliamo tali componenti attivate grazie all' interazione, nonché gli adeguamenti reciproci svolti dagli insegnanti e dagli allievi per farsi capire e per capirsi, i supporti visivi che utilizzano, il ruolo che giocano le due lingue.

Parole chiave: Pratiche di introduzione alla scrittura, bambini sordi, bilinguismo, lettura interattiva, costruzione del saper leggere/scrivere. 


\title{
Which literacy practices for Deaf Children?
}

\begin{abstract}
This article describes some literacy practices introduced in a bilingual classroom for deaf children. We will complete the rooted concepts of bilingual programs in the education of these children with concepts from the field of emergent literacy studies in the general population, and among others, from the field of a didactic microgenesis. An Interactive Reading session will serve as an illustration of the components of the literacy knowledge that emerge in the classroom discourse. In this complex didactic situation, we apprehend these components enabled with/in the interaction, as well as reciprocal adjustments made by teachers and pupils to negotiate their understanding of the story. We will also examine the supports they use, and the role the two languages play in this interaction.
\end{abstract}

Key words: Literacy practices, deaf children, bilingual education, interactive reading, literacy knowledge construction 